\title{
11 INDETERMINISM AND PLURALISM IN NATURE: FROM SCIENCE TO PHILOSOPHY AND THEOLOGY
}

\author{
Claudia E. Vanney
}

The relationship between determinism and freedom has been one of the main concerns in philosophy throughout history. Many philosophers have opposed freedom (or chance) to necessity (or causality) when discussing whether freedom is present or not in the connection between processes and their results, as a way to differentiate between necessary (or causal) processes and free (or random) processes. Different definitions and notions have been used in the debates over determinism. On the one hand, the terms contingency, randomness, emergency, uncertainty, free will and freedom seem to point to an indeterministic position on reality. On the other hand, the notions of necessity, causation, lawlikeness, predictability and fatalism seem to suggest a deterministic stance. But does science properly distinguish between these notions? If so, how does it do this? Furthermore, how does philosophy contribute towards the elucidation of such distinctions? Does a deterministic or an indeterministic world view affect theological thought?

In the deterministic view, everything that has happened (past), everything that happens (present) and everything that will happen (future) is already determined, conditioned or established. During the classical and medieval periods, determinism had a metaphysical connotation, derived from the principle of universal causation. In modern times, however, deterministic conceptions laid their theoretical foundations in scientific knowledge and were associated with the notions of prediction and law-likeness. Paradigmatic mechanistic determinism regarded the world as a great clockwork-like system. Since time is the independent variable in the dynamical equations of mechanics, it is possible to describe every mechanical process as a temporal succession of different possible states. Thus, mechanistic determinism conceived that physical systems inexorably evolve from an initial state.

During the twentieth century, the scientific world view distanced itself considerably from the deterministic image of the clockwork-like world, thus 
assuming new modalities. On the one hand, the development of chaos theory turned out to be an insurmountable obstacle for those who aspired to anticipate a univocal prediction of all future states in all real systems. ${ }^{1}$ On the other hand, the consolidation of quantum mechanics has called for a revision of classic determinism insofar as it introduces randomness in the fundamental strata of reality. ${ }^{2}$ Thereafter, a new indeterministic paradigm was progressively introduced in different areas of physics and other sciences, changing the vision of the world and also affecting the specific objectives of science. In the emergence of quantum mechanics, some authors saw the death of determinism. Others preferred to circumvent the problem by adopting an instrumentalist position. Finally, a minority assumed that quantum mechanics is an incomplete theory and postulated the possibility of a broader formulation that would recover determinism.

The deterministic proposals are still in force, however, and should not be identified with classical mechanistic determinism. Nowadays, determinism assumes different meanings depending on whether it is applied to the universe as a whole (regularities of nature), to our knowledge (predictability) or to the dynamical equation of movement (laws of science). Indeed, several contemporary authors consider determinism to be only a property of scientific theories. Nevertheless, setting the precise way in which the different theories are related to one another is still an open problem. Discussions over the relationship between micro- and macro-evolutions in physics and biology have also revealed the tension between reductionist and anti-reductionist comprehensions of science. While reductionists believe that determinism/indeterminism is resolved at the fundamental level, anti-reductionists accept the possibility of a simultaneous coexistence of deterministic and indeterministic descriptions in different strata of reality.

Far from being a resolved issue, the debate over the determinism/indeterminism of the natural world has opened new perspectives on philosophical discussions. On the one hand, the distinction between determinism and predictability has led to deeper research into the relationship between ontological and gnoseological realms. On the other hand, the multiple descriptions proposed by contemporary science cannot avoid the question of the cognitive status of the various scientific formulations and the possibility of a coexistence of deterministic and indeterministic views. Furthermore, many authors suggest the necessity of an ontological indeterminism in order to admit a divine action in nature; this is a proposal with broad implications in natural theology.

In this chapter, I shall present some philosophical ideas suggested by Leonardo Polo which could shed new light on the aforementioned questions. Polo (19262013) was a Spanish philosopher still largely unknown in English-speaking academic circles but whose ideas have awakened an increasing interest over recent years in several academic environments in Spain and also in Latin America. On this continent, Polo participated in fruitful academic activity - particularly in 
Mexico, Colombia and Peru - and supported incipient intellectual groups in a number of different countries.

Polo's thought is not anchored in any philosophical school exclusively; it builds upon many previous philosophical proposals which merit further interpretation. Polo holds the conviction that philosophy, for the same reason that it does not exhaust its first inspiration, is a kind of knowledge that always allows further development. Polo's regard for science, as with his profound openness towards transcendence, makes his philosophy a useful platform for dealing with the different topics of science and religion. His proposals have not yet been studied in-depth but they offer an original and inspirational starting point for future studies. With Polo in mind, I propose that the recognition of a variety of cognitive acts represents a suitable approach to the illumination of the determinism/indeterminism debate of the natural world. In my opinion, Polo's theory of knowledge not only enables a more precise formulation of the terms used in this discussion but also establishes a broader framework for a more fruitful interdisciplinary dialogue.

\section{The Emergence of a New Indeterministic Paradigm}

The development of complex sciences during the twentieth century has put mechanistic determinism to the test. The coexistence - in chaotic systems - of non-lineal deterministic laws, together with the uncertainty of the initial conditions of the physical system, has made it impossible to have univocal predictions of the temporal evolution of each of the many particles in a real system. Although we can estimate the temporal evolution of the initial imprecisions of different variables, the final uncertainties depend largely on the characteristics of the equations of movement. When the equations which govern the temporal evolution of a physical system are linear, the predictions about their evolution are kept within a limited range. In complex systems, however, the uncertainty about the initial state of the system makes it impossible to predict the temporal evolution of each of the particles within the system. Given that, in complex systems the movement of particles is governed by equations which are very sensitive to the initial conditions, the trajectories that follow two points which are initially very close, diverge exponentially (and not linearly) with the passing of time. ${ }^{3}$

Nevertheless, it is still possible to maintain determinism in chaotic systems, but this requires a particular interpretation. ${ }^{4}$ Since future states in a complex system can be statistically predicted from a holistic perspective, the apparently random macroscopic processes can be interpreted as the answers given by underlying microscopic, deterministic laws that are responsible for restoring the univocal temporal dependence between the states. If we consider that the only function of statistics is to allow for the treatment of very complex systems with 
a great degree of freedom, probability may be seen as an expression of our ignorance of perfectly deterministic processes that result from an infinite number of unobservable elements. ${ }^{5}$

When we consider determinism/indeterminism as an intrinsic property of nature we are faced with an ontological problem. From the study of the temporal evolution of chaotic systems, however, a new interpretation of indeterminism which highlights gnoseological aspects may also emerge. From this viewpoint, indeterminism is not a property of nature but a manifestation that we lack sufficient information of in order to determine the dynamical evolution of certain systems. That is, gnoseological indeterminism primarily reinforces the limitation of our cognitive capacity. This indeterminacy can be compatible with an ontological determinism, but this determinism remains unknowable for us.

This broadening of perspective made it possible to separate the notions of law-likeness and predictability, which are inherent properties of the mechanistic determinism. ${ }^{6}$ The deterministic laws govern the dynamical evolution of complex systems, so that each state is rigidly determined by the previous state. The evolution of each particle, however, can only be predicted with some degree of probability. The difficulty of predicting future states of the particles which compose the system can be interpreted in two ways. First, as the revelation of an ontological indeterminism in nature. Second, as a measurement of our ignorance about the perfect deterministic process which each individual microscopic particle, whose behaviour we know only through statistical procedures, follows.

\section{Ontological, Gnoseological and Scientific Determinism}

Until relatively recently, the consideration of a constant and univocal succession of events that constitute the history of the universe was always implicit in deterministic conceptions, without making any reference to methodological or epistemological issues. During the last century, however, the notion of determinism was also addressed from the perspective of the analysis of language and today many authors consider determinism to be a property of scientific theories, understanding the latter as a deductively closed set of statements of a formal language. Nevertheless, as the term determinism applies to diverse entities, I think that it is useful to distinguish between the different meanings of this concept. ${ }^{7}$ Firstly, ontological determinism considers the predicate 'deterministic' to be applicable to the universe as a whole. Scientific laws governing the behaviour of real systems are regularities inscribed in the ontological realm. Thus, ontological determinism is a property of nature. Secondly, gnoseological determinism assumes that the predicate 'deterministic' applies to our knowledge of the dynamical evolution of a system. This meaning identifies determinism with 
predictability. Consequently, gnoseological determinism is a property of our knowledge. Finally, scientific determinism considers the predicate 'deterministic' applicable to the dynamical equations that univocally govern the evolution of physical systems. From this standpoint, the aim of science is to describe real systems through deterministic equations. Thus, scientific determinism is a property of scientific theories.

Nevertheless, if we attempt to draw ontological conclusions about a physical system from an exclusively formal analysis, some difficulties are bound to emerge. The laws of physics describe the time evolution of physical systems through a system of differential equations which governs the behaviour of several variables over time. It is usually thought that dynamical equations of motion are deterministic when a given value of independent variables univocally fixes the dynamical evolution of a physical system in any given state. In this way, the deterministic character of a given scientific theory, or the lack of it, will usually be associated with the possibility of finding unique solutions for dynamical equations: if these possible solutions are not unique, there will be no determinism. ${ }^{8}$

In accordance with this criterion, some authors have tried to classify diverse theories into deterministic or indeterministic categories. ${ }^{9}$ This is by no means an easy task to accomplish, since each of the different theories includes different concepts - such as 'system' or 'state' - which are frequently not defined with the necessary precision. Hence, even within each theory there will be an open space to formulate legitimately a notion of determinism in different ways, thus requiring an interpretative discernment so as to choose the best formulation in each case. ${ }^{10}$ In order to ascertain the determinism or indeterminism in a given scientific theory, it is necessary to adopt a metatheoretical epistemological perspective, and this calls for moving towards an in-depth study of the kind of reasoning behind scientific theories. For example, the measurement problem in quantum theory seems to disclose very clearly the indeterminism of this theory. Since the deterministic approaches have always assumed the existence of a univocal connection between the properties of a physical system throughout time, the measurement problem reveals a peculiarity of quantum systems: they do not possess all their properties defined in each instant. Nevertheless (and despite the fact that quantum physics has a large number of successful predictions), the understanding of the theory is not yet clear enough, since there are several different interpretations of quantum mechanics. ${ }^{11}$

Nowadays, most interpretations of quantum physics favour an indeterministic framework. Even though there are also new deterministic interpretations of quantum mechanics, such as Bohm's, ${ }^{12}$ in 1964 Bell's theorem proved that it is not possible that a deterministic and local theory of hidden variables which reproduced the correlations that Bohm predicted could exist. ${ }^{13}$ Bell's theoretical arguments were empirically confirmed by Aspect some years later, thus bringing 
relevant elements against a deterministic and local interpretation of quantum results. ${ }^{14}$ It has not yet been resolved, however, whether the most recent interpretations of quantum physics favour an ontological or an epistemological indeterminism, since quantum mechanics could either introduce an ontological indeterminism in microphysics, or quantum probabilities may simply be a sign of our ignorance of the quantum world.

\section{Reduction}

Even current scientific theories with wide acceptance among researchers usually contain philosophical problems regarding their foundations which are related to topics of interpretation - a paradigmatic example is quantum mechanics or inter-theoretic articulation. For example, we have seen that highly unstable dynamical systems are deterministic in a microscopic description, but they are indeterministic in a macroscopic one. In the debate between determinism and indeterminism in the natural world, the dilemma between a reductionist and an anti-reductionist understanding of science is also present. Reductionist positions usually attribute determinism/indeterminism to the most fundamental level. Anti-reductionists, in turn, accept a simultaneous coexistence of different theoretic formulations in different strata of reality. For this second position, the different scientific theories do not offer a unitary image of the world. ${ }^{15}$

In the first half of the twentieth century, the reductionist programme intended to reduce all scientific theories to only one, regarded as fundamental. ${ }^{16} \mathrm{~W}$ ithin this programme, it is possible to distinguish different kinds of reductionism: (1) semantic reductionism (the language of the reduced scientific field is translated into the language of the reducing field); (2) inter-theoretic reductionism (the laws of the reduced theory are deduced from the reducing theory); (3) methodological reductionism (the method of the reducing theory is the privileged one) ${ }^{17}$ Besides, these reductionisms are usually supported by an ontological reductionism (the reducing theory contains the field of reality of the reduced theory).

The links between different physical theories usually involve a mathematical limit or, inversely, coarse-graining techniques. ${ }^{18}$ Due to some 'successful' cases of reduction in the field of physics, reductionism was uncritically assumed on several occasions. The reduction of thermodynamics to statistical mechanics is a paradigmatic case. Even 'successful' cases, however, continue to pose open problems: thermodynamics contains fundamental laws which are not t-invariant. Thus, how could we explain irreversibility with reversible theories or how could an irreversible world be depicted adequately within a reversible theory? ${ }^{19}$ Quantum mechanics also offers a good example: the complexity of the problem of decoherence shows that to deduce classical mechanics from quantum mechanics implies something more than the mere application of a mathematical limit. ${ }^{20}$ 
The discussion about the relationship between micro- and macro-evolution soon moved from physics to biology. Progress in molecular biology also led to new reductionist proposals, which sought to reduce all biological explanations to the domain of molecular biology. Classical genetic reductionism, like Monod's, paved the way for indeterministic perspectives in biology by introducing chance in its various forms, as a generator of diversity and a mechanism of evolution, among others. ${ }^{21}$ For later determinism, however, it is genes, together with environmental conditions, that completely determine the morphology and the behaviour of the phenotypes. ${ }^{22}$ Nevertheless, just as there are no biological laws that could be reduced to the laws of molecular biology, genes and DNA do not adequately satisfy the criteria of reduction either. At present, there are some anti-reductionist biologists who consider the explanations of macrobiology appropriate and autonomous enough so as to require neither correction, nor completion, nor any additional explanations at the molecular level. ${ }^{23}$

\section{Cognitive Pluralism}

The contemporary debate on the determinism/indeterminism of the natural world has opened up new perspectives on philosophical reflection. On the one hand, the distinction between determinism and predictability permits the exploration of how the ontological and the gnoseological domains relate to one another. On the other hand, the plurality of descriptions put forward by contemporary science raises the question of the cognitive status of different scientific formulations. Leonardo Polo's theory of knowledge could allow access to both topics. Between 1984 and 1996, Polo carried out important gnoseological clarifications which can be found in the four volumes of his Curso de Teoria del Conocimiento. ${ }^{24}$ In Polo's view, knowledge has two dimensions in perfect agreement: one is the cognitive act (methodic dimension) and the other one is the content known (thematic dimension). So, knowledge is an act that is unitarily thematic or a theme that is unitarily an act. ${ }^{25}$ In other words, no theme appears without accounting for the intellectual method that leads to its consideration, and there is no intellectual act which does not delimit its theme in a clear way. Polo also distinguishes several kinds of cognitive acts, and he points out that no cognitive dimension can be considered absolute. In my opinion, this methodic-thematic pluralism not only relates the gnoseological (methodic) to the ontological (thematic) realms without confusing them, but also paves the way for establishing the cognitive status of the different scientific disciplines avoiding reduction. ${ }^{26}$

Extra-mental reality is regarded as different from the known object. What is thought is admittedly non-real, since it has no nature of its own apart from being an aspectual reference. The object is extra-mental reality that is thought 
in act, whereas the act of thinking is immanent. Due to its intentionality, the object thought 'does not properly need to be. ${ }^{27}$ That is, extra-mental principles are not like objects or reality. Objectification is not false, but it grants only a limited access to what is real, since the order of what is real is not intentional but extra-mental. Objectifications provide a valid yet insufficient knowledge of reality. They are inevitably multiform because they are aspectual. Since they consist of different ways of referring to reality they entail truth, but this is restricted to the aspect they refer to. Nonetheless, the aspectual plurality of intentional knowledge does not equal the real thing, since it is not possible to reconstruct reality by composing different intentionally known aspects. This is why numerous descriptions of different scientific theories do not offer a unitary vision of the cosmos, which some reductionists aspire to. The unification of reality in terms of totality is generated by thought, which introduces uniqueness.

Therefore, if ontology aspires to have some knowledge of the true principles of reality and not just an intentional knowledge, a cognitive method different from intentional objectification is required. Polo states that it is possible to realize that reality is plural and diverse within itself if an appropriate cognitive method is used. He proposes a new method to access knowledge of the real principles of the universe, which I shall refer to in more detail below. By applying one dimension of his method, and following Aristotle, he recognizes different principles or causes in the world: in the universe there is contingency (material cause), determination (formal cause), dynamism (efficient cause) and order (final cause). But these principles do not exist in isolation, they only exist in co-causality. ${ }^{28}$

\section{Indeterminism and Divine Action}

The discussion of determinism/indeterminism in the natural world is not only a concern for epistemology and philosophy of science; it also has strong implications for natural theology. Many authors have emphasized the fact that processes in the world have involved, at all stages of its history, an interplay between chance and necessity. For some of them, novelty emerges at the edge of chaos, where order and disorder connect without destroying one another. ${ }^{29} \mathrm{~A}$ recent research programme called 'Scientific Perspectives on Divine Action' studied how contemporary science points to a kind of metaphysical space which can allow for divine agency in the world. ${ }^{30}$ According to researchers of this programme, in selecting the laws of nature, God chooses specific laws with very remarkable properties. Thus, the laws allow not only for chance events but also for the genuine emergence of complexity in nature - an emergence which requires these laws but goes far beyond a mere unfolding of their consequences. 
Nevertheless, the need for an ontological indeterminism in order to admit a divine action in nature is not at all evident. Some scholars, such as William E. Carroll and Ignacio Silva, have pointed out the importance of distinguishing between divine action and natural action. If we assume that God acts in nature through 'gaps of indetermination' or through regions where natural causality is not well defined, we are employing a univocal notion of causality which hinders the metaphysical distinction between divine causality and created causality.

When we consider that God's actions are like those of natural causes - causes in the natural order - some difficulties are likely to arise. If God's causal status is reduced to the causal status of any other cause, divine action loses its provident character: it is hard to understand how a cause that is just one among others could guide the created world towards its final destination. In order to avoid a univocal understanding of causality it is possible, for example, to resort to the classical notions of primary and secondary causality. ${ }^{31}$ God is the first cause of the effect, while agents are secondary causes. So, on the one hand, if we consider the causal power thanks to which the action is executed, God is the cause of the action of natural agents. On the other hand, the natural agent considered in itself is the immediate cause of its effect. That is, an analogical notion of causality, instead of a univocal understanding, can refer both to divine and created causality, leaving room for the necessary differences that preserve God's transcendence and a provident divine action.

I think that it is also possible to address this question from Polo's perspective. I have already mentioned that his gnoseological clarification sheds light on the range of scientific knowledge. Polo's philosophical proposal is inspired by the purpose of transcending the predominance of conceptual objectivity in knowledge. That is, the discovery that 'uniqueness' and 'totality' belong exclusively to the mental realm and are therefore erroneously applied to extra-mental reality. The awareness of the limits of objectuality triggered a far-reaching philosophical enterprise, and Polo proposed a method of his own for philosophy, which he called the abandonment of the mental limit. Polo decided to look for different ways of abandoning that limit in all topics proper to philosophy. ${ }^{32}$ The discrimination of the different modes in which objectuality puts being aside makes it possible to access both metaphysics and anthropology, while also establishing an important distinction between both of them. I shall come back to this distinction later. Polo states that, in order to have knowledge of causality, a proper intellectual method is needed, one that is different from objectification. Thus, in contrast with what the programme 'Scientific Perspectives on Divine Action' maintains, it would not be possible for Polo to access knowledge of God by means of an objectifying knowledge. The 'idea' of God would be formulated by means of objectification but this does not grant knowledge of God as a primary being. 
Polo also distinguishes several real principles, because extra-mental principles do not all belong to just one kind. We can distinguish the 'principles of principled things' (principios de las cosas principiadas) - such as the material or formal cause - from the act of being as the principle of existence, which Polo calls persistence (persistencia). Philosophy of nature deals with the former, whereas metaphysics studies the latter. The aforementioned adjustment between method and theme also demands different cognitive methods for these philosophical disciplines. So, the abandonment of the mental limit in its first dimension is the methodological way which Polo proposes in order to access knowledge of a very wide range of topics, proper to metaphysics and natural theology, such as the awareness of extra-mental existence and of the created condition, among others. In particular, Polo's perspective underlines the transcendence of God. ${ }^{33}$

The four Aristotelian causes are, for Polo, real principles in the predicamental order. They are, furthermore, the analysis of one first transcendental principle: persistence. But persistence is different from the origin. The divine act of being is original activity. What is original has always been, whereas what is originated has a beginning. Created being is persistence, whereas the Origin is uncreated being. The creature is a caused cause, whereas God is uncaused and creator of the cause. To know extra-mental being - abandoning the limit of thought - means to notice the radical dependence of the universe with respect to God; its strict character as a creature.

Even though it is not possible to give more details of Polo's proposal in these pages, I think that what has been said is enough to maintain that - according to Polo - science, ontology and natural theology approach the study of nature through different cognitive methods and, therefore, they result in different kinds of knowledge. Nevertheless, the methodic-thematic discrimination suggested by Polo does not entail the claim that there would be no communication between these disciplines. On the contrary, since these disciplines deal with different dimensions, they shed light on one and the same problem from different perspectives. So, scientific discoveries can inspire fruitful developments in philosophy of nature and natural theology. In an interdisciplinary dialogue, however, only a consideration of the cognitive range of the different statements can avoid naïve concordisms (for example, between science and religion) and extrapolations that lack precision beyond their own field. In my opinion, if different disciplines are to engage in a suitable critical dialogue, it is imperative to have a clear epistemological frame. 


\section{Indeterminism, Uncertainty, Unpredictability, Freedom}

The distinction between ontological, gnoseological and scientific determinism has already been mentioned above. The same distinction between different levels of analysis could also refer to indeterminism: ontological indeterminism describes an intrinsic property of nature; gnoseological indeterminism indicates a characteristic of our knowledge; and scientific indeterminism refers to the laws of science. In order to avoid an incorrect assimilation of concepts, however, I suggest reserving the use of indeterminism for the ontological realm. Uncertainty, in turn, could refer more properly to gnoseological statements. Finally, unpredictability is a notion which has a full meaning in scientific contexts. Besides, in accordance with Polo's proposal, we could also say that the indeterminism of the natural world confirms its contingency (understood as a real principle); that uncertainty indicates that the human cognitive power is not absolute, and that unpredictability points at the limitations of objectifying knowledge in general and of scientific knowledge in particular.

The problem of determinism unavoidably also affects the question of freedom, which opens up an anthropological perspective. Classical positions fall into compatibilism and incompatibilism, ${ }^{34}$ even if the magnitude of such a distinction has been questioned. ${ }^{35}$ While various compatibilists claim that a deterministic system leaves room for free will, ${ }^{36}$ incompatibilists deny such a statement. ${ }^{37}$ Among these, libertarians say that free will is real but presupposes indeterminism in the physical world. ${ }^{38}$ Others adopt a hard deterministic position, according to which determinism is true and so excludes the possibility of free acts. ${ }^{39}$ It is not true, however, that every indeterministic position leaves free will uncompromised. If, for example, the fixation of quantum indeterminacies were due to random movements, human action would not have its origin in a genuinely free decision..$^{40}$ Leonardo Polo did not participate in this debate, but his transcendental anthropology also offers a new perspective of analysis. ${ }^{41}$ Even though in philosophical tradition, 'transcendental' usually means what is most universal or transcategorial, Polo understands this concept as an act of being. In this context, he points out that the (act of) being studied by metaphysics cannot be the (act of) being studied by anthropology. This is because metaphysics deals with being as a principle or foundation, and this meaning of being excludes freedom, since a dependent and founded freedom is something contradictory. Salvador Piá, one of Polo's early followers, explains:

Polo proposes a transcendental distinction between metaphysics (understood as the study of the cosmos) and anthropology (understood as the study of the human being). These two sciences study distinct types of acts of being; the former studies the act of being of the physical universe (that is, the act of persistence), while the latter studies the act of being of the human person (that is, the act of co-existence). ${ }^{42}$ 
Thereby, if we follow the abandonment of the mental limit in order to know the human person, an anthropological extension of the traditional doctrine of the transcendentals becomes necessary. Polo proposes that freedom is a transcendental of human person - that is to say - convertible with the personal act of being. ${ }^{43}$ Since indeterminism of physical reality and human freedom refer to real and transcendental principles of different kinds, they are neither identified nor mutually implied in a direct way.

\section{Conclusion}

The question of determinism/indeterminism in nature, in the past century, has materialized in a novel way in physics (quantum mechanics, theory of chaos); biology (theory of evolution, biology of development, system biology); and in the neurosciences (the problem of freedom). Through science, a new indeterministic paradigm has been gradually introduced to philosophical and theological reflections, changing the world vision and influencing the specific aims of scientific research.

To address these and similar issues, it seems desirable to cross systematically the borders of what is traditionally considered scientific, philosophical or theological, in order to face the specific challenge of attaining interdisciplinary understanding and a common technical vocabulary that promotes a dialogue between different disciplines. Nevertheless, this dialogue must be developed with care. On the one hand, it is important to avoid an incorrect transposition of the ontological, gnoseological and epistemological levels, because that could lead to confusion. On the other hand, in order to avoid reductionism, we should not consider any of these levels as unique or absolute. The methodological proposal of the abandonment of the mental limit, which I have very briefly presented in this chapter, entails a deep renunciation of any form of monism and of the kind of uniqueness that results from resting at an objectification. But if we accept that operative knowledge - which is abstractive and objectifying - is neither the only nor the supreme kind of knowledge, it is possible - according to Polo - to leave uniqueness in search of other ways of accessing physical and metaphysical reality and, above all, what is intrinsically human.

In my opinion, Polo's philosophical proposal offers a radical anti-reductionist answer: science's objectifying way of thinking is not the only possible way of knowing. Knowledge is wider than thought, since thought is a specific kind of knowledge. Polo's philosophy offers many suggestive insights but, since his writings still contain many unexplored aspects, it is still too early to pass a judgement on its capacity to participate in contemporary debates with clarifying answers that also encourage its continuation. 